\title{
Complete Monotonicity of Functions Connected with the Exponential Function and Derivatives
}

\author{
Chun-Fu Wei ${ }^{1,2}$ and Bai-Ni Guo ${ }^{2}$ \\ ${ }^{1}$ State Key Laboratory Cultivation Base for Gas Geology and Gas Control, Henan Polytechnic University, \\ Jiaozuo, Henan 454010, China \\ ${ }^{2}$ School of Mathematics and Informatics, Henan Polytechnic University, Jiaozuo, \\ Henan 454010, China \\ Correspondence should be addressed to Chun-Fu Wei; mathwcf@163.com
}

Received 25 January 2014; Accepted 20 March 2014; Published 15 April 2014

Academic Editor: Beong In Yun

Copyright (C) 2014 C.-F. Wei and B.-N. Guo. This is an open access article distributed under the Creative Commons Attribution License, which permits unrestricted use, distribution, and reproduction in any medium, provided the original work is properly cited.

Some complete monotonicity results that the functions $\pm 1 /\left(e^{ \pm t}-1\right)$ are logarithmically completely monotonic, and that differences between consecutive derivatives of these two functions are completely monotonic, and that the ratios between consecutive derivatives of these two functions are decreasing on $(0, \infty)$ are discovered. As applications of these newly discovered results, some complete monotonicity results concerning the polylogarithm are found. Finally a conjecture on the complete monotonicity of the above-mentioned ratios is posed.

\section{Introduction and Main Results}

Throughout this paper, we denote the set of all positive integers by $\mathbb{N}$.

Recently, the following problem was posed in $[1$, page 569]. For $t \neq 0$ and $k \in \mathbb{N}$, determine the numbers $a_{k, i-1}$ for $1 \leq i \leq k$ such that

$$
\frac{1}{\left(1-e^{-t}\right)^{k}}=1+\sum_{i=1}^{k} a_{k, i-1}\left(\frac{1}{e^{t}-1}\right)^{(i-1)}
$$

This problem, among other things, was answered in [1] by eight identities. Two of the eight identities may be recited as follows.

Theorem A (see [1, Theorem 2.1]). For $i \in\{0\} \cup \mathbb{N}$, one has

$$
\left(\frac{1}{e^{t}-1}\right)^{(i)}=\sum_{m=1}^{i+1} \frac{\lambda_{i, m}}{\left(e^{t}-1\right)^{m}}
$$

where

$$
\begin{gathered}
\lambda_{i, m}=(-1)^{i}(m-1) ! S(i+1, m), \\
S(i, m)=\frac{1}{m !} \sum_{\ell=1}^{m}(-1)^{m-\ell}\left(\begin{array}{c}
m \\
\ell
\end{array}\right) \ell^{i}, \quad 1 \leq m \leq i,
\end{gathered}
$$

are Stirling numbers of the second kind.

Theorem B (see [1, Theorem 2.3]). For $i, k \in \mathbb{N}$ with $1 \leq i \leq k$, the coefficients $a_{k, i-1}$ defined in (1) may be calculated by

$$
a_{k, i-1}=(-1)^{i^{2}+1} M_{k-i+1}(k, i),
$$




$$
M_{j}(k, i)=\left|\begin{array}{cccc}
\frac{1}{(i-1) !}\left(\begin{array}{c}
k \\
i
\end{array}\right) & S(i+1, i) & \cdots & S(i+j-1, i) \\
\frac{1}{i !}\left(\begin{array}{c}
k \\
i+1
\end{array}\right) & S(i+1, i+1) & \cdots & S(i+j-1, i+1) \\
\frac{1}{(i+1) !\left(\begin{array}{c}
k \\
i+2
\end{array}\right)} & 0 & \cdots & S(i+j-1, i+2) \\
\vdots & \vdots & \ddots & \vdots \\
\frac{1}{(i+j-2) !}\left(\begin{array}{c}
k \\
i+j-1
\end{array}\right) & 0 & \cdots & S(i+j-1, i+j-1)
\end{array}\right| .
$$

For more information, please refer to $[2,3]$ and related references therein.

Stimulated by results obtained in [1], as mentioned above, we are interested in two functional sequences:

$$
\begin{aligned}
& F_{i}(t)=(-1)^{i}\left(\frac{1}{e^{t}-1}\right)^{(i)}, \\
& G_{i}(t)=(-1)^{i}\left(\frac{1}{1-e^{-t}}\right)^{(i)},
\end{aligned}
$$

where $t \in(0, \infty)$ and $i \in\{0\} \cup \mathbb{N}$, and we firstly discover in this paper the following results.

Theorem 1. For $i \in\{0\} \cup \mathbb{N}$, the functions $F_{i}(t)$ and $G_{i}(t)$ are completely monotonic on $(0, \infty)$. More strongly, the functions $F_{0}(t)$ and $G_{0}(t)$ are logarithmically completely monotonic on $(0, \infty)$.

For $i, k \in\{0\} \cup \mathbb{N}$, the functions $F_{i}(t)$ and $G_{i}(t)$ satisfy

$$
\begin{aligned}
& F_{i}^{(k)}(t)=(-1)^{k} F_{i+k}(t), \\
& G_{i}^{(k)}(t)=(-1)^{k} G_{i+k}(t) .
\end{aligned}
$$

Theorem 2. For given $i \in\{0\} \cup \mathbb{N}$, the differences

$$
\begin{aligned}
& \mathfrak{F}_{i}(t)=F_{i+1}(t)-F_{i}(t), \\
& \mathfrak{G}_{0}(t)=G_{1}(t)-G_{0}(t)+1, \\
& \mathfrak{G}_{i+1}(t)=G_{i+2}(t)-G_{i+1}(t)
\end{aligned}
$$

are completely monotonic functions on $(0, \infty)$. In particular, the sequences $F_{i}(t)$ and $G_{i}(t)$ are increasing with respect to $i$ for $t \in(0, \infty)$; that is, the inequalities

$$
F_{i}(t)<F_{i+1}(t), \quad G_{i}(t)<G_{i+1}(t)
$$

are valid for all $i \in\{0\} \cup \mathbb{N}$ and $t \in(0, \infty)$.

Theorem 3. For given $i \in\{0\} \cup \mathbb{N}$, the ratios

$$
\mathscr{F}_{i}(t)=\frac{F_{i+1}(t)}{F_{i}(t)}, \quad \mathscr{G}_{i}(t)=\frac{G_{i+1}(t)}{G_{i}(t)}
$$

are decreasing on $(0, \infty)$, with

$$
\lim _{t \rightarrow \infty} \mathscr{F}_{i}(t)=1, \quad \lim _{t \rightarrow \infty} \mathscr{G}_{0}(t)=0, \quad \lim _{t \rightarrow \infty} \mathscr{G}_{i+1}(t)=1 .
$$

In order to further show the importance and significance of the two functional sequences in (6), we secondly give several applications of Theorems 1 to 3 in Section 4.

Finally, we pose a conjecture on the complete monotonicity of the functions $\mathscr{F}_{i}(t)$ and $\mathscr{G}_{i}(t)$ defined in $(10)$.

\section{Two Definitions and a Lemma}

Now we list definitions of the completely monotonic and the logarithmically completely monotonic functions, which just now appeared in Theorems 1 and 2, and recite a lemma, which is needed to prove Theorem 3.

Definition 4 (see $[4,5]$ ). A function $q(x)$ is said to be completely monotonic on an interval $I$ if $q(x)$ has derivatives of all orders on $I$ and $(-1)^{n} q^{(n)}(x) \geq 0$ for $x \in I$ and $n \geq 0$.

The noted Hausdorff-Bernstein-Widder theorem [5, page 161, Theorem 12b] says that a necessary and sufficient condition that $f(x)$ should be completely monotonic for $0<x<$ $\infty$ is that

$$
f(x)=\int_{0}^{\infty} e^{-x t} \mathrm{~d} \alpha(t),
$$

where $\alpha(t)$ is nondecreasing and the integral converges for $0<x<\infty$. In other words, a function $f$ defined on $(0, \infty)$ is completely monotonic on $(0, \infty)$ if and only if it is a Laplace transform. For more information on the theory of completely monotonic functions, please refer to [4, Chapter XIII], [5, Chapter IV], and the newly published monograph [6]. This means that it is useful to confirm the complete monotonicity of functions.

Definition 5 (see $[7,8]$ ). A positive function $f(x)$ is said to be logarithmically completely monotonic on an interval $I \subseteq \mathbb{R}$ if it has derivatives of all orders on $I$ and its logarithm $\ln f(x)$ satisfies $(-1)^{k}[\ln f(x)]^{(k)} \geq 0$ for $k \in \mathbb{N}$ on $I$.

It has been proved that any logarithmically completely monotonic function on $I$ is also completely monotonic on $I$, 
but not conversely. For more information on this class of functions, please refer to [7-9] and [10, Section 1.3] and closely related references therein. This shows that it is helpful to prove the logarithmically complete monotonicity of functions.

Lemma 6 (see [11, Lemma 2.2]). Suppose that $a_{k}, b_{k}>0$ and that $\left\{u_{k}(t)\right\}$ is a sequence of positive and differentiable functions such that the series

$$
\sum_{k=0}^{\infty} a_{k} u_{k}^{(\ell)}(t), \quad \sum_{k=0}^{\infty} b_{k} u_{k}^{(\ell)}(t), \quad \ell=0,1,
$$

converge absolutely and uniformly over compact subsets of $[0, \infty)$.

(1) If the logarithmic derivative $u_{k}^{\prime}(t) / u_{k}(t)$ forms an increasing sequence of functions and if $a_{k} / b_{k}$ decreases (resp., increases), then

$$
f(t)=\frac{\sum_{k=0}^{\infty} a_{k} u_{k}(t)}{\sum_{k=0}^{\infty} b_{k} u_{k}(t)}
$$

decreases (resp., increases) for $t \geq 0$.

(2) If the logarithmic derivative $u_{k}^{\prime}(t) / u_{k}(t)$ forms a decreasing sequence of functions and if $a_{k} / b_{k}$ decreases (resp., increases), then the function $f(t)$ increases (resp., decreases) for $t \geq 0$.

\section{Proofs of Main Results}

Now we start out to prove our theorems.

Proof of Theorem 1. Since

$$
\begin{aligned}
& F_{0}(t)=G_{0}(t)-1=\sum_{\ell=1}^{\infty} e^{-\ell t}, \\
& F_{i}(t)=G_{i}(t)=\sum_{\ell=1}^{\infty} \ell^{i} e^{-\ell t}
\end{aligned}
$$

for $i \geq 1$, the functions $F_{i}(t)$ and $G_{i}(t)$ for $i \in\{0\} \cup \mathbb{N}$ are completely monotonic on $(0, \infty)$.

Taking the logarithms of the functions $F_{0}(t)$ and $G_{0}(t)$ and differentiating yield

$$
\begin{aligned}
& {\left[\ln F_{0}(t)\right]^{\prime}=\frac{\mathrm{d}}{\mathrm{d} t}\left(\ln \frac{1}{e^{t}-1}\right)=-\frac{e^{t}}{e^{t}-1}=-\left[F_{0}(t)+1\right]} \\
& {\left[\ln G_{0}(t)\right]^{\prime}=\frac{\mathrm{d}}{\mathrm{d} t}\left(\ln \frac{1}{1-e^{-t}}\right)=-F_{0}(t) .}
\end{aligned}
$$

Consequently, by definition of logarithmically completely monotonic functions and the above obtained complete monotonicity of the function $F_{0}(t)$, it is ready to deduce the logarithmically complete monotonicity of $F_{0}(t)$ and $G_{0}(t)$ on $(0, \infty)$.

The formulas in (7) can be straightforwardly verified. The proof of Theorem 1 is complete.
Proof of Theorem 2. A simple computation yields

$$
\begin{aligned}
& F_{i+1}(t)-F_{i}(t) \\
& =(-1)^{i+1}\left[\left(\frac{1}{e^{t}-1}\right)^{(i+1)}+\left(\frac{1}{e^{t}-1}\right)^{(i)}\right] \\
& =(-1)^{i+1}\left[\left(\sum_{\ell=1}^{\infty} e^{-\ell t}\right)^{(i+1)}+\left(\sum_{\ell=1}^{\infty} e^{-\ell t}\right)^{(i)}\right] \\
& =(-1)^{i+1}\left[\sum_{\ell=1}^{\infty}(-1)^{i+1} \ell^{i+1} e^{-\ell t}+\sum_{\ell=1}^{\infty}(-1)^{i} \ell^{i} e^{-\ell t}\right] \\
& =\sum_{\ell=2}^{\infty}(\ell-1) \ell^{i} e^{-\ell t} \\
& >0, \\
& (-1)^{n}\left[F_{i+1}(t)-F_{i}(t)\right]^{(n)}=\sum_{\ell=2}^{\infty}(\ell-1) \ell^{n+i} e^{-\ell t}>0
\end{aligned}
$$

for $n \in \mathbb{N}$. By definition, the difference $\mathfrak{F}_{i}(t)=F_{i+1}(t)-F_{i}(t)$ for $i \in\{0\} \cup \mathbb{N}$ is completely monotonic on $(0, \infty)$.

From the first equalities in (15), respectively, it follows that the functions

$$
\begin{gathered}
\mathfrak{G}_{0}(t)=G_{1}(t)-G_{0}(t)+1=F_{1}(t)-F_{0}(t), \\
\mathfrak{G}_{i+1}(t)=G_{i+2}(t)-G_{i+1}(t)=F_{i+2}(t)-F_{i+1}(t)
\end{gathered}
$$

are all completely monotonic on $(0, \infty)$.

The inequalities in (9) follow from the positivity of $\mathfrak{F}_{i}(t)$ and $\mathfrak{G}_{i}(t)$ for $i \in \mathbb{N}$. The proof of Theorem 2 is complete.

Proof of Theorem 3. It is easy to see that

$$
\begin{aligned}
\mathscr{F}_{i}(t) & =-\frac{\left[1 /\left(e^{t}-1\right)\right]^{(i+1)}}{\left[1 /\left(e^{t}-1\right)\right]^{(i)}}=-\frac{\left(\sum_{k=1}^{\infty} e^{-k t}\right)^{(i+1)}}{\left(\sum_{k=1}^{\infty} e^{-k t}\right)^{(i)}} \\
& =-\frac{\sum_{k=1}^{\infty}(-1)^{i+1} k^{i+1} e^{-k t}}{\sum_{k=1}^{\infty}(-1)^{i} k^{i} e^{-k t}}=\frac{\sum_{k=1}^{\infty} k^{i+1} e^{-k t}}{\sum_{k=1}^{\infty} k^{i} e^{-k t}} .
\end{aligned}
$$

Let $a_{k}=k^{i+1}, b_{k}=k^{i}$, and $u_{k}(t)=e^{-k t}$. Then $a_{k} / b_{k}=k$ is an increasing sequence and $u_{k}^{\prime}(t) / u_{k}(t)=-k$ form a decreasing sequence. By Lemma 6 , it follows that the function $\mathscr{F}_{i}(t)$ is decreasing on $(0, \infty)$.

Taking $t \rightarrow \infty$ at the very ends of (19) shows that

$$
\begin{aligned}
\lim _{t \rightarrow \infty} \mathscr{F}_{i}(t) & =\lim _{t \rightarrow \infty} \frac{\sum_{k=1}^{\infty} k^{i+1} e^{-k t}}{\sum_{k=1}^{\infty} k^{i} e^{-k t}} \\
& =\lim _{t \rightarrow \infty} \frac{1+\sum_{k=2}^{\infty} k^{i+1} e^{(1-k) t}}{1+\sum_{k=2}^{\infty} k^{i} e^{(1-k) t}}=1 .
\end{aligned}
$$

The first limit in (11) thus follows.

Making use of the second relation in (15) yields $\mathscr{G}_{i}(t)=$ $\mathscr{F}_{i}(t)$ for $i \in \mathbb{N}$. Accordingly, the function $\mathscr{G}_{i}(t)$ has the same monotonicity and the same limit for $t \rightarrow \infty$ as $\mathscr{F}_{i}(t)$ does for 
$i \in \mathbb{N}$. As a result, the function $\mathscr{G}_{i}(t)$ is decreasing on $(0, \infty)$ and the third limit in (11) is valid for $i \in \mathbb{N}$.

A straightforward computation gives

$$
\mathscr{G}_{0}(t)=\frac{1}{e^{t}-1}=F_{0}(t)
$$

which obviously tends to 0 as $t \rightarrow \infty$ and apparently decreases on $(0, \infty)$. The proof of Theorem 3 is complete.

\section{Some Applications}

We recall from [12] that the polylogarithm $\operatorname{Li}_{s}(z)$ is the function

$$
\operatorname{Li}_{s}(z)=\sum_{k=1}^{\infty} \frac{z^{k}}{k^{s}}
$$

defined for all $s \in \mathbb{C}$ and over the open unit disk $|z|<1$ in the complex plane $\mathbb{C}$. Its definition on the whole complex plane then follows uniquely via analytic continuation.

For given $i \in\{0\} \cup \mathbb{N}$, since $F_{i}(t)=\sum_{\ell=1}^{\infty} \ell^{i} e^{-\ell t}$, we observe that

$$
F_{i}(t)=\mathrm{Li}_{-i}\left(e^{-t}\right), \quad G_{i+1}(t)=\operatorname{Li}_{-(i+1)}\left(e^{-t}\right) .
$$

Therefore, considering (15), Theorems 1 to 3 can be used to find some properties of the polylogarithm $\operatorname{Li}_{s}(z)$ as follows.

Theorem 7. For $i \in\{0\} \cup \mathbb{N}$, the polylogarithm $\mathrm{Li}_{-i}\left(e^{-t}\right)$ is completely monotonic with respect to $t \in(0, \infty)$. More strongly, the polylogarithm $\mathrm{Li}_{0}\left(e^{-t}\right)$ is logarithmically completely monotonic with respect to $t \in(0, \infty)$.

For $i, k \in\{0\} \cup \mathbb{N}$, the polylogarithm $\mathrm{Li}_{-i}\left(e^{-t}\right)$ satisfies

$$
\left[\mathrm{Li}_{-i}\left(e^{-t}\right)\right]^{(k)}=(-1)^{k} \mathrm{Li}_{-(i+k)}\left(e^{-t}\right) \text {. }
$$

Theorem 8. For given $i \in\{0\} \cup \mathbb{N}$, the differences

$$
\begin{aligned}
& \mathfrak{\mho}_{i}(t)=\mathrm{Li}_{-(i+1)}\left(e^{-t}\right)-\mathrm{Li}_{-i}\left(e^{-t}\right), \\
& \mathfrak{G}_{i}(t)= \begin{cases}\operatorname{Li}_{-i}\left(e^{-t}\right)-\mathrm{Li}_{0}\left(e^{-t}\right)+1, & i=0, \\
\mathfrak{F}_{i+1}(t), & i \geq 1,\end{cases}
\end{aligned}
$$

are completely monotonic functions on $(0, \infty)$. In particular, the polylogarithm sequence $\mathrm{Li}_{-i}\left(e^{-t}\right)$ is increasing with respect to $i$ for given $t \in(0, \infty)$; that is, the inequality

$$
\operatorname{Li}_{-i}\left(e^{-t}\right)<\operatorname{Li}_{-(i+1)}\left(e^{-t}\right)
$$

is valid for all $i \in\{0\} \cup \mathbb{N}$ and $t \in(0, \infty)$.

Theorem 9. For given $i \in\{0\} \cup \mathbb{N}$, the ratios

$$
\begin{gathered}
\mathscr{F}_{i}(t)=\frac{\operatorname{Li}_{-(i+1)}\left(e^{-t}\right)}{\mathrm{Li}_{-i}\left(e^{-t}\right)}, \\
\mathscr{G}_{i}(t)= \begin{cases}\mathscr{F}_{i}(t), & i \geq 1, \\
\frac{\mathrm{Li}_{-i}\left(e^{-t}\right)}{1+\mathrm{Li}_{0}\left(e^{-t}\right)}, & i=0,\end{cases}
\end{gathered}
$$

are decreasing on $(0, \infty)$, with

$$
\lim _{t \rightarrow \infty} \mathscr{F}_{i}(t)=1, \quad \lim _{t \rightarrow \infty} \mathscr{G}_{0}(t)=0, \quad \lim _{t \rightarrow \infty} \mathscr{G}_{i+1}(t)=1 .
$$

Furthermore, by the above (logarithmically) complete monotonicity in Theorems 7 and 8 and by some complete monotonicity properties of composite functions, we can obtain the complete monotonicity of functions involving the polylogarithm $\mathrm{Li}_{-i}(1 / t)$ for $i \in\{0\} \cup \mathbb{N}$ as follows.

Theorem 10. The following complete monotonicity is valid.

(1) The polylogarithm $\operatorname{Li}_{0}(1 / t)=1 /(t-1)$ is logarithmically completely monotonic with respect to $t \in(1, \infty)$.

(2) For $i \in\{0\} \cup \mathbb{N}$, the polylogarithm $\mathrm{Li}_{-i}(1 / t)$ is completely monotonic with respect to $t \in(1, \infty)$.

(3) For given $i \in\{0\} \cup \mathbb{N}$, the differences

$$
\begin{gathered}
\mathfrak{P}_{i}(t)=\mathrm{Li}_{-(i+1)}\left(\frac{1}{t}\right)-\mathrm{Li}_{-i}\left(\frac{1}{t}\right), \\
\mathfrak{Q}_{i}(t)= \begin{cases}\mathrm{Li}_{-i}\left(\frac{1}{t}\right)-\mathrm{Li}_{0}\left(\frac{1}{t}\right)+1, & i=0, \\
\mathfrak{P}_{i+1}(t), & i \geq 1,\end{cases}
\end{gathered}
$$

are completely monotonic functions on $(1, \infty)$.

Proof. The second item of [13, Theorem 5] tells us that if $h^{\prime}(x)$ is completely monotonic on an interval $I$ and $f(x)$ is logarithmically completely monotonic on the domain $h(I)$, then the composite function $f \circ h(x)=f(h(x))$ is logarithmically completely monotonic on $I$. It is easy to verify that the derivative of $\ln t$ is $1 / t$ and completely monotonic on $(0, \infty)$. Combining these conclusions with the logarithmically complete monotonicity of $\operatorname{Li}_{0}\left(e^{-t}\right)$ in Theorem 7 yields that the polylogarithm $\operatorname{Li}_{0}(1 / t)=1 /(t-1)$ is logarithmically completely monotonic with respect to $t \in(1, \infty)$.

In [14, page 83], it was given that if $f$ and $g$ are functions such that $f(g(x))$ is defined on $(0, \infty)$ and if $f$ and $g^{\prime}$ are completely monotonic, then $x \mapsto f(g(x))$ is also completely monotonic on $(0, \infty)$. Replacing $f(t)$ by $\operatorname{Li}_{-i}\left(e^{-t}\right)$ and $g(t)$ by $\ln t$ and making use of the complete monotonicity of $\mathrm{Li}_{-i}\left(e^{-t}\right)$ in Theorem 7 lead to the complete monotonicity of the polylogarithm $\mathrm{Li}_{-i}(1 / t)$.

The leftover proofs are similar to the above arguments. The proof of Theorem 10 is complete.

Remark 11. To the best of our knowledge, the above (logarithmically) complete monotonicity results concerning the polylogarithm are new. This shows us the importance of the two functional sequences in (6) and the significance of Theorems 1 to 3 . 


\section{A Conjecture}

By Theorem 1 and the fact that any logarithmically completely monotonic function on $I$ is also completely monotonic on $I$, it is clear that the functions

$$
\begin{gathered}
\mathscr{G}_{0}(t)=\mathscr{F}_{0}(t)-1=\frac{1}{e^{t}-1}=F_{0}(t), \\
\mathscr{G}_{1}(t)=\mathscr{F}_{1}(t)=1+\frac{2}{e^{t}-1}=1+2 F_{0}(t)
\end{gathered}
$$

are all completely monotonic on $(0, \infty)$. This motivates us to pose the following conjecture.

Conjecture 12. For given $i \in\{0\} \cup \mathbb{N}$, the functions $\mathscr{F}_{i}(t)$ and $\mathscr{G}_{i}(t)$ defined in (10) or (27) are completely monotonic on $(0, \infty)$.

\section{Conflict of Interests}

The authors declare that there is no conflict of interests regarding the publication of this paper.

\section{Acknowledgments}

The authors are grateful to the anonymous referees for their valuable comments on and careful corrections to the original version of this paper. The authors appreciate Professor Dr. Feng Qi in China for his valuable contribution to this paper. Chun-Fu Wei was partially supported by the NNSF under Grant no. 51274086 of China, by the Ministry of Education Doctoral Foundation of China-Priority Areas under Grant no. 20124116130001, and by the State Key Laboratory Cultivation Base for Gas Geology and Gas Control under Grant no. WS2012A10 at Henan Polytechnic University, China.

\section{References}

[1] B.-N. Guo and F. Qi, "Some identities and an explicit formula for Bernoulli and Stirling numbers," Journal of Computational and Applied Mathematics, vol. 255, pp. 568-579, 2014.

[2] F. Qi, "Explicit formulas for computing Euler polynomials in terms of the second kind Stirling numbers ," http://arxiv.org/ abs/1310.5921.

[3] A.-M. Xu and Z.-D. Cen, "Some identities involving exponential functions and Stirling numbers and applications," Journal of Computational and Applied Mathematics, vol. 260, pp. 201-207, 2014.

[4] D. S. Mitrinović, J. E. Pečarić, and A. M. Fink, Classical and New Inequalities in Analysis, vol. 61, Kluwer Academic Publishers, 1993.

[5] D. V. Widder, The Laplace Transform, Princeton University Press, Princeton, NJ, USA, 1946.

[6] R. L. Schilling, R. Song, and Z. Vondraček, Bernstein Functions, vol. 37 of de Gruyter Studies in Mathematics, De Gruyter, Berlin, Germany, 2010.

[7] R. D. Atanassov and U. V. Tsoukrovski, "Some properties of a class of logarithmically completely monotonic functions," Comptes Rendus de l'Académie Bulgare des Sciences, vol. 41, no. 2, pp. 21-23, 1988.
[8] C. Berg, "Integral representation of some functions related to the gamma function," Mediterranean Journal of Mathematics, vol. 1, no. 4, pp. 433-439, 2004.

[9] F. Qi and Q. M. Luo, "Bounds for the ratio of two gamma functions: from Wendel's asymptotic relation to Elezovic-GiordanoPecaric's theorem," Journal of Inequalities and Applications, vol. 2013, article 542, 20 pages, 2013.

[10] F. Qi and Q.-M. Luo, "Bounds for the ratio of two gamma functions: from Wendel's and related inequalities to logarithmically completely monotonic functions," Banach Journal of Mathematical Analysis, vol. 6, no. 2, pp. 132-158, 2012.

[11] S. Koumandos and H. L. Pedersen, "On the asymptotic expansion of the logarithm of Barnes triple gamma function," Mathematica Scandinavica, vol. 105, no. 2, pp. 287-306, 2009.

[12] Wikipedia, “The Free Encyclopedia," http://en.wikipedia.org/ wiki/Polylog.

[13] F. Qi and B.-N. Guo, "Some logarithmically completely monotonic functions related to the gamma function," Journal of the Korean Mathematical Society, vol. 47, no. 6, pp. 1283-1297, 2010.

[14] S. Bochner, Harmonic Analysis and the Theory of Probability, California Monographs in Mathematical Sciences, University of California Press, Berkeley, Calif, USA, 1955. 


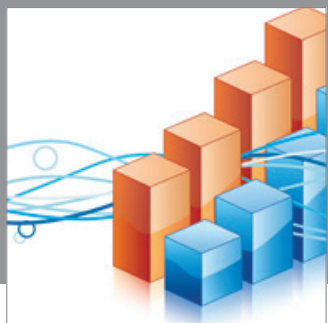

Advances in

Operations Research

mansans

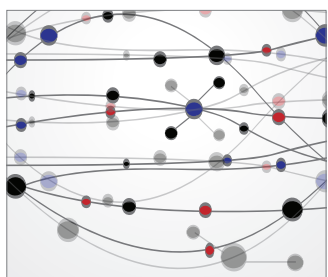

The Scientific World Journal
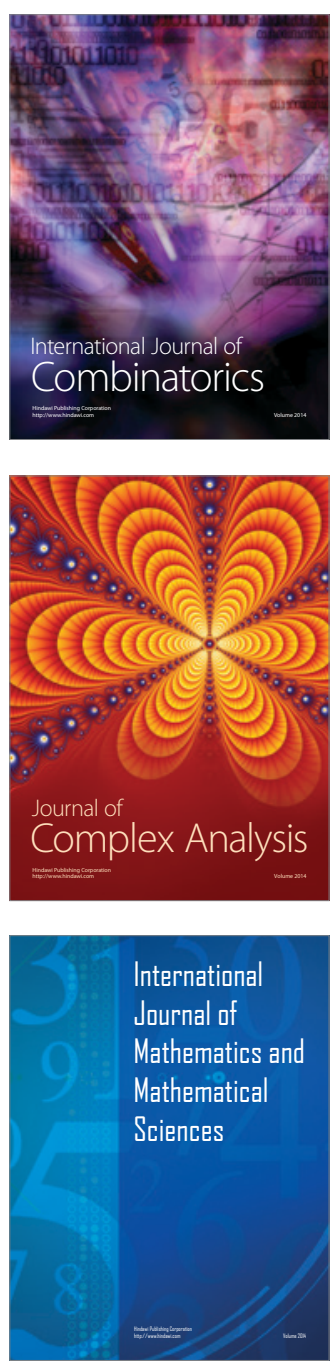
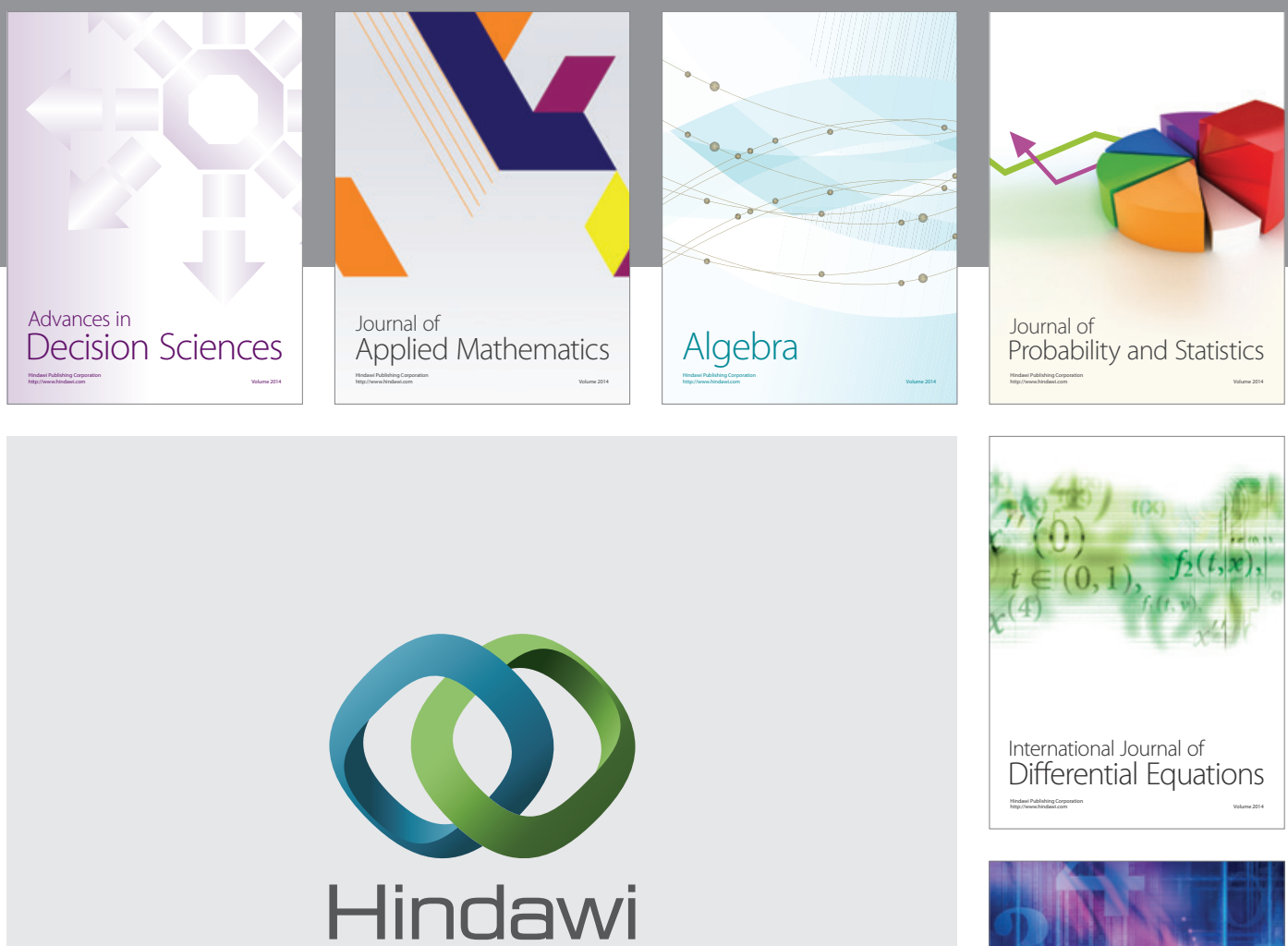

Submit your manuscripts at http://www.hindawi.com
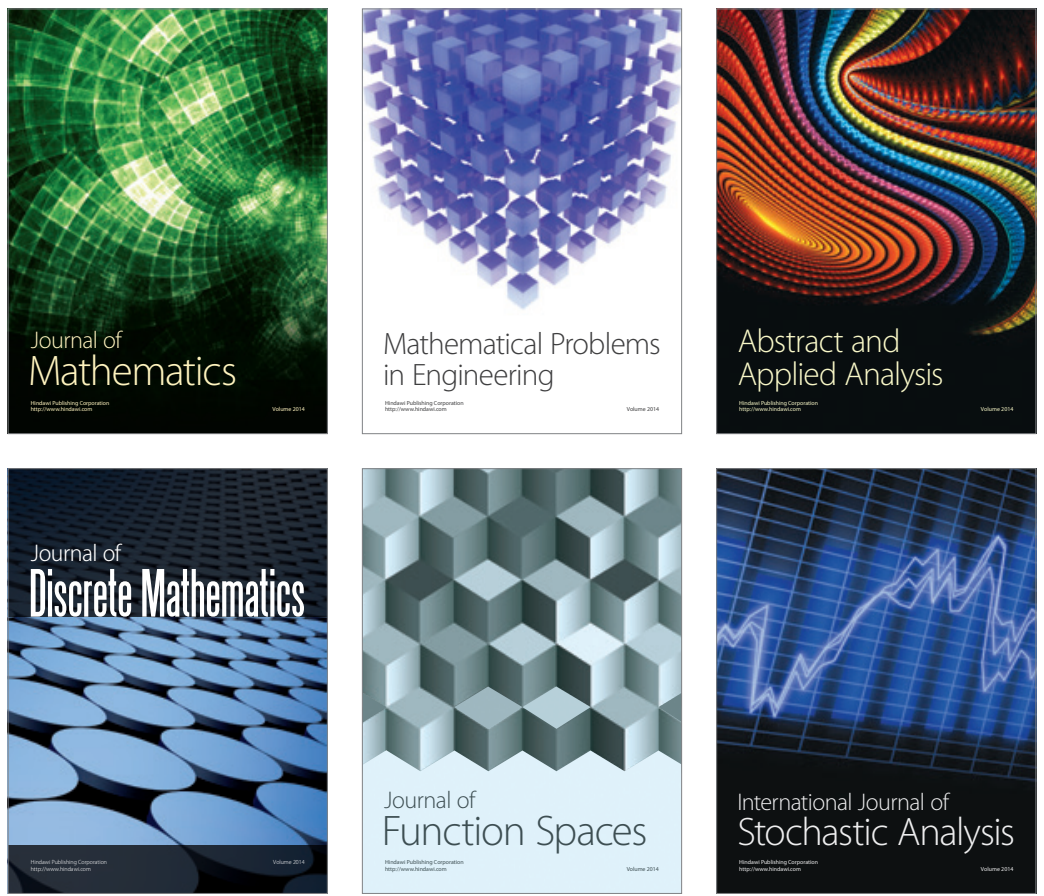

Journal of

Function Spaces

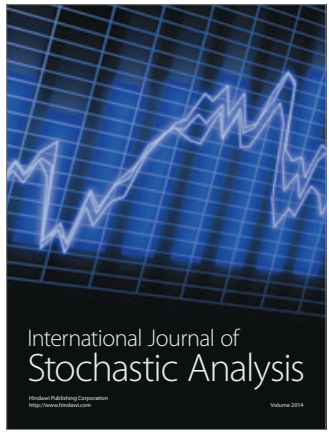

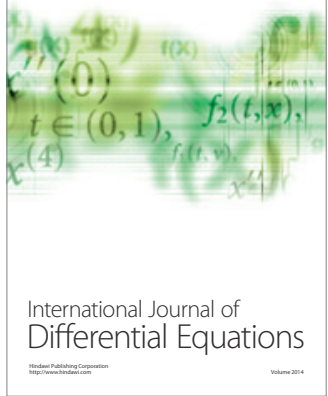
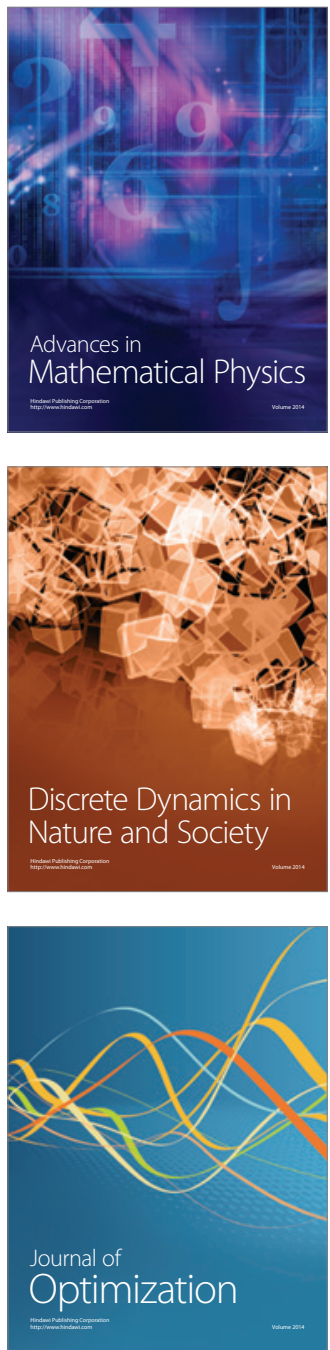